\title{
$\mathrm{K}-\mathrm{R} A S$ mutation in the screening, prognosis and
}

\section{treatment of cancer}

The potential use of K-RAS mutation as a cancer screening biomarker has been investigated for many years. Numerous associations between K-RAS mutation and various cancers have been established, but these associations have not been translated into effective, cost-efficient cancer screening strategies. This lack of progress may be due to the existence of K-RAS mutation in nontumor tissues and/or using detection, rather than quantitation, of K-RAS mutation as the endpoint for cancer risk categorization. K-RAS mutation appears to be a useful prognostic biomarker for colon cancer. Recent progress toward sensitive and quantitative mutation characterization and the successful use of K-RAS mutation in a personalized medicine approach to targeted biological therapy selection are likely to re-direct and expand the use of K-RAS mutation as a cancer biomarker in the near future.

\section{KEYWORDS: biomarker carcinogenesis mutation detection oncogene personalized medicine}

Developing informative cancer biomarkers is essential to achieve early detection of tumors, to better stratify patients in terms of prognosis and to optimize therapy for individual cancer patients. Investigation in this area is vigorous and a variety of technologies and end points are being developed. Mutation is one type of biomarker being investigated, with mutation in the $\mathrm{K}-R A S$ gene the archetype mutational cancer biomarker. An extensive literature describes the tumor prevalence and the cellular consequences of $\mathrm{K}-R A S$ mutation. The $\mathrm{K}-R A S$ mutant phenotype provides a strong rationale for using $\mathrm{K}-R A S$ mutation as a biomarker of cancer risk.

\section{K-RAS function, mutant phenotype \& prevalence}

Several lines of reasoning have led to the expectation that somatic mutations should be useful cancer biomarkers. Cancer is considered to be a genetic disease and somatic mutations in oncogenes and tumor suppressor genes are viewed as necessary events in carcinogenesis. Initiating mutations, therefore, are expected to be present at the earliest stages of tumor development and persist during progression. The extent to which $\mathrm{K}-R A S$ mutations are found in human tumors is exceeded only by mutations in the $p 53$ gene $(\sim 50 \%)$, which are distributed over many codons. For K-RAS, it has been established that a few codons encompass the majority of reported mutations. Specifically, hotspot point mutations in codons 12 and 13 are found in significant percentages of human tumors.
Normal K-RAS protein function and the effects of particular K-RAS base substitution mutations on K-RAS protein function are well described. Accurate post-translational processing of K-RAS (including farnesylation or guanylguanylation of a C-terminal CAAX box) is needed for membrane anchoring [1]. K-RAS is a GTP-binding protein that functions in transducing the signal from ligand-specific receptor binding (including dimeric epidermal growth factor receptor [EGFR]-type molecules: EGFR-EGFR, EGFR-HER2, EGFR-HER3 or EGFR-HER4) through intracellular signaling cascades, eventually impacting nuclear transcription [2]. Mutation at particular sites in $\mathrm{K}-R A S$ favors the active, GTP-bound form of RAS, which leads to aberrant intracellular signaling through the RAF-MEK-ERK kinase and the PI3K-Akt pathways. Because the RASRAF-MEK-ERK kinase pathway regulates normal cell-cycle control, mutation in K-RAS causes unregulated cell proliferation [3]. Aberrant signaling through the PI3K-Akt pathway activates a cascade of anti-apoptotic and prosurvival signals [2]. In addition, mutant $\mathrm{K}-R A S$ increases tissue factor and $V_{E G F}{ }_{189}$ mRNA levels, suggesting it also plays a role in angiogenesis and tumor progression [4].

The $\mathrm{K}-R A S$ mutant phenotype is complex, in that different $\mathrm{K}-R A S$ mutations confer slightly different selective advantages, which in turn are applicable to particular tissue types [3]. Differences in $\mathrm{K}-R A S$ mutant phenotype have been associated with different amino acid substitutions at
Barbara L Parsons

\& Fanxue Meng

${ }^{\dagger}$ Author for correspondence:

US Food and Drug

Administration, National

Center for Toxicological

Research, Division of Genetic

\& Reproductive Toxicology,

HFT-120, 3900 NCTR Rd.

Jefferson, AR 72079, USA

Tel.: +18705437946 ;

Fax: +18705437393 ;

barbara.parsons@fda.hhs.gov 
codon 12 [5]. Janssen et al. showed that mouse embryo fibroblasts expressing normal levels of $\mathrm{K}-R A S^{\mathrm{G} 12 \mathrm{D}}$ (codon 12 GGT to GAT) are partially transformed, demonstrate enhanced proliferative properties, lack contact inhibition, and can initiate tumors in animals [6]. A transgenic model specifically expressing $\mathrm{K}-R A S^{\mathrm{G} 12 \mathrm{D}}$ in a stem cell compartment of mouse colon was found to recapitulate human colon tumor development in terms of low tumor yield and latency time [6]. Furthermore, this occurred in the absence of $A P C$ mutation, consistent with the idea that $\mathrm{K}-R A S$ is important for colon tumor initiation, just as it has demonstrated importance in pancreatic tumor initiation. The relevance of particular K-RAS mutations in different tissues can be inferred from their prevalence in various tumor/lesion types (Table 1). Such information is now easily accessible and searchable through the Catalogue of Somatic Mutations in Cancer (COSMIC) database [7,101].

\section{$\mathrm{K}-\mathrm{RAS}$ mutation as a biomarker in cancer screening}

Development of good cancer screening strategies has been one of the most intractable areas of cancer research, but an area with great potential for public health impact. A successful cancer screening strategy needs to combine several critical elements:

- Analysis of a readily available specimen source;

- Cancer biomarker validation (an established association with disease state);

\section{Table 1. Prevalence of K-RAS mutations in human tumors.}

\begin{tabular}{|lll|}
\hline Tumor type & $\begin{array}{l}\text { Positive samples per } \\
\text { number analyzed }\end{array}$ & $\begin{array}{l}\text { Percent K-RAS } \\
\text { mutant }\end{array}$ \\
\hline Pancreas & $2842 / 4773$ & $60 \%$ \\
\hline Biliary & $474 / 1478$ & $32 \%$ \\
\hline Large intestine & $5513 / 17126$ & $32 \%$ \\
\hline Small intestine & $61 / 300$ & $20 \%$ \\
\hline Lung & $1981 / 11401$ & $17 \%$ \\
\hline Endometrium & $251 / 1750$ & $14 \%$ \\
\hline Ovary & $243 / 1696$ & $14 \%$ \\
\hline Prostate & $74 / 919$ & $8 \%$ \\
Cervix & $49 / 645$ & $7 \%$ \\
\hline Stomach & $147 / 2345$ & $6 \%$ \\
\hline $\begin{array}{l}\text { Liver } \\
\text { Testis }\end{array}$ & $22 / 365$ & $6 \%$ \\
\hline "Data collected from the Catalogue of Somatic Mutations in Cancer database [7,101]. Data current as \\
of April 28, 2009. Tumor types with sample numbers less than 100 or percent mutated less than 5\% \\
were not included.
\end{tabular}

- A methodology with which to measure the biomarker.

These three elements need to be considered throughout the development of potential biomarkers.

$\mathrm{K}-R A S$ mutation has been examined in a variety of clinical specimens. Generally, studies have compared the prevalence of $\mathrm{K}-R A S$ mutation in body fluids from cancer patients to the prevalence observed in healthy control subjects as a first step toward validating the use of $\mathrm{K}-R A S$ mutation in cancer screening. Clinical samples that have been analyzed for $\mathrm{K}-\mathrm{R} A S$ mutation include $\mathrm{blood} /$ plasma/serum, urine, stool, pancreatic juice, sputum and bronchoalveolar lavage fluid. Using denaturing high-pressure liquid chromatography combined with mutant allele-specific amplification (DHPLC-MASA), Lilleberg et al. report $100 \%$ concordance in terms of the mutations (including $\mathrm{K}-R A S$ ) measured in the tumor and plasma samples of colorectal cancer (CRC) patients [8]. K-RAS mutation was detected in ten out of $18(56 \%)$ plasma samples from patients with a $\mathrm{K}-R A S$ mutant tumor or polyp [9]. Using a quantitative beaming-based approach, Diehl et al. found $50 \%$ of plasma samples had the same measurable mutation as the tumor from colon cancer patients [10].

Plasma samples from lung and pancreatic cancer patients also are being analyzed for $\mathrm{K}-R A S$ mutant DNA. Using PCR in combination with a restriction fragment length polymorphism (RFLP) methodology, Kimura et al. detected $\mathrm{K}-R A S$ mutation in the plasma of five out of 25 (20\%) non-small-cell lung cancer (NSCLC) patients [11]. A meta-analysis of studies investigating the prevalence of $\mathrm{K}-R A S$ mutation in the circulating DNA of lung cancer patients reports that the mutation is detected in $43 \%$ of cases but in only $0.8 \%$ of healthy controls [12]. Using a peptide nucleic acid-PCR clamp, Luo et al. detected $\mathrm{K}-R A S$ mutation in the plasma of 19 out of $24(79.2 \%)$ pancreatic cancer patients [13]. Using an enriched-nested PCR, Jiao et al. detected K-RAS mutation in the plasma of 25 out of $77(32.5 \%)$ pancreatic cancer patients and concluded the mutation was associated with a history of smoking [14].

$\mathrm{K}-R A S$ mutation has been detected in relatively low molecular weight DNA isolated from the urine of CRC patients $[15,16]$. K-RAS mutation was detected in 15 out of $18(83.3 \%)$ urine samples from patients with a $\mathrm{K}-R A S$ mutant tumor or polyp [9]. Su et al. characterized the amount of $\mathrm{K}-R A S$ mutant DNA, as well as 
the ratio of mutant to wild-type (WT) K-RAS sequence in urine samples, and reported an $83 \%$ concurrence between detection of mutant DNA in urine and within the colon tumor [17].

A few studies have analyzed sputum for K-RAS mutation. Using PCR-RFLP analysis, Destro et al. found the same K-RAS mutation in sputum samples as in patients' NSCLCs [18]. Using PCR and single-stranded conformational polymophism (PCR-SSCP), K-RAS mutation was detected in the epithelial cells collected from the sputum of seven out of 15 (46.7\%) lung cancer patients [19].

Despite a large number of retrospective investigational studies, only a few studies have evaluated $\mathrm{K}-R A S$ mutation as a prospective marker for tumor development and the results from these studies have been only marginally encouraging. Using a method that could detect mutant DNA at a level of 3-12\% of wild-type DNA, K-RAS mutation was measured in the serum of individuals who were then followed for 6.3 years [20]. Serum K-RAS mutations were detected in 13 out of 1098 subjects (1.2\%) and six of these developed a cancer during the follow-up period. Significantly, five of the K-RAS-positive cancers were bladder cancers (five out of 137 cases, odds ratio of 4.25) [20]. However, serum K-RAS mutations also were detected in seven out of 713 $(0.01 \%)$ subjects who did not develop a cancer during the follow-up period. With respect to pancreatic cancer, a large prospective multicenter trial on the frequency of K-RAS in pancreatic juice and bile had a sensitivity of $38 \%$ and a specificity of $90 \%$ for the diagnosis of pancreatic cancer, with $\mathrm{K}-R A S$ mutation also being detected in benign disease, chronic pancreatitis (considered a premalignant condition) and normal pancreas [21].

A significant effort has been directed toward the detection of K-RAS mutation in DNA isolated from stool samples, or in colonocytes isolated from stool. This effort is summarized in a recent review by Loktionov [22]. K-RAS mutations were among the genetic lesions detected by a commercially available stool-based DNA screening test for CRC (the PreGen-Plus ${ }^{\mathrm{TM}}$ test released by Labcorp, NC, USA in 2003 and later discontinued). Results obtained using stool DNA-based screening (of which K-RAS mutation was just a part) were compared with those obtained on 4404 asymptomatic patients undergoing colonoscopy [23]. Stool-based screening detected 16 of 31 cancers $(51.6 \%$ sensitivity) with a specificity of $94.4 \%$. Given its sensitivity and the assay's cost, PreGen-Plus was not considered sufficiently cost-effective for a general colon cancer screening test [24,25]. Stool DNA-based screening has also been investigated as an approach to confirm positive results in fecal occult blood tests (FOBT) [26]. DNA was successfully amplified from $87.2 \%$ of FOBT cards. Combining detection of K-RAS mutation with positive FOBT improved the positive predicitive value for malignancy from 17.7 to $60.0 \%$. Using a quantitative beaming-based approach, Diehl et al. found $92 \%$ of stool DNA samples had the same measurable mutation as the tumor from colon cancer patients [10]. Efforts to develop DNAbased colon cancer screening continue, with a focus on improving DNA isolation from stool or from the mucocellular layer on the surface of the rectal mucosa [22,27,28].

Since mutation in the human KRAS2 protooncogene is almost by definition a cancer-relevant marker, rarely is the tumor-relevance of $\mathrm{K}-R A S$ mutation discussed. However, it must be recognized that there are numerous reports of $\mathrm{K}-R A S$ mutation detection in normal tissues, benign pathological tissues and plasma samples. For example, one study found K-RAS mutation in $30 \%$ of plasma samples from noncancer controls [29]. K-RAS mutation has been detected in normal colonic mucosa of colon cancer patients [30]. Using a sensitive, quantitative allele-specific competitive blocker-PCR (ACBPCR) approach, K-RAS codon 12 GAT mutation was detected in colonic mucosa samples from individuals without colon cancer and in all mucosa samples from colon cancer patients [31]. $\mathrm{K}-R A S$ mutation was detected in the serum of $30 \%$ of 40 noncancer hospital controls [32]. Several studies have reported the detection of $\mathrm{K}-R A S$ mutation in pancreatic juice from patients with chronic pancreatitis [21]. In fact, when pancreatic juice is tested, $\mathrm{K}-R A S$ mutations are found in $20-30 \%$ of patients with chronic pancreatitis [33]. Importantly, Shi et al. showed that by quantifying levels of K-RAS mutation they could establish a cut-off level above which there was a statistically-significant association between $\mathrm{K}-R A S$ mutation and pancreatic cancer, and no association with chronic pancreatitis [34]. Therefore, detection of K-RAS mutation does not necessarily denote the presence of a malignancy. Indeed, the presence of $\mathrm{K}-R A S$ mutation in the absence of cancer may explain why removing K-RAS from the PreGenPlus genetic battery increased the specificity of the test from 91 to $100 \%$ [35]. Given the limited progress in applying K-RAS mutation as a cancer screening biomarker and the data indicating 
$\mathrm{K}-R A S$ detection cannot be equated with disease, future research should focus on using sensitive approaches that quantify $\mathrm{K}-R A S$ mutation in blood or urine and investigate using cut-off values of $\mathrm{K}-R A S$ mutation as triggers for more rigorous cancer screening.

\section{K-RAS mutation as a prognostic biomarker}

Prognostic biomarkers are needed for several reasons: to decide whether a more or less aggressive treatment or no treatment is warranted, to aid in patient follow-up and to identify patients who have relapsed or are about to relapse. Because a prognostic biomarker is not applied to very large numbers of healthy individuals, it doesn't need the very high specificity and cost effectiveness that is necessary for a screening biomarker. Compared to the development of a screening biomarker, development of a prognostic biomarker to stratify risk in a cancer patient population is a less demanding task.

A significant body of research indicates $\mathrm{K}-R A S$ mutational status has prognostic value, whether measured in colorectal tumors or the plasma samples of patients with a $\mathrm{K}-R A S$ positive tumor. The large multicenter Kirsten Ras in Colorectal Cancer Collaborative group (RASCAL) studies found no association between $\mathrm{K}-R A S$ mutation and tumor stage, but showed that detection of a $\mathrm{K}-R A S$ mutation in a patient's tumor significantly increases the risk of tumor recurrence and death [36-38]. Also, a significant association between $\mathrm{K}-R A S$ codon 12 mutation and poor 5-year survival has been demonstrated [39]. In particular, the K-RAS $S^{\mathrm{G} 12 \mathrm{~V}}$ mutation (codon 12 GGT to GTT) has been associated with poor prognosis, including a $50 \%$ increased risk of relapse or death [37,40]. This base substitution apparently causes significant changes in Ras protein function, in terms of its GTP-binding affinity and GTPase activity [40]. In addition, transversion mutations in $\mathrm{K}-R A S$ codon 12 (including TGT and GTT) are over-represented in smokers as compared with never smokers [41]. Interestingly, the recent study by Winder et al. suggests for the first time that $\mathrm{K}-R A S$ mutations (other than codon 12 valine) in colorectal carcinoma are associated with increased patient survival relative to patients with wild-type tumors [40].

More recent studies have addressed the question of whether detection of $\mathrm{K}-R A S$ mutation in free circulating DNA can predict tumor recurrence. Using a PCR-single strand conformation polymorphism analysis, Wang et al. showed serum K-RAS mutation, in conjunction with $A P C$ and $p 53$ mutation, was associated with a higher postoperative metastasis/recurrence rate [42]. Cancer-related, 24-month survival was reached by five out of nine (56\%) Dukes' stage D patients without circulating $\mathrm{K}-R A S$ mutation but none out of seven $(0 \%)$ Dukes' stage $D$ patients with circulating $\mathrm{K}-R A S$ mutation [43]. In a multicenter prospective study, $\mathrm{K}-R A S$ mutation (but not $p 53$ mutation or $\mathrm{p}^{16^{\mathrm{INK}} 4 \mathrm{~A}}$ methylation) in plasma DNA was significantly associated with accelerated patient relapse [44]. After 3 years, approximately $78 \%$ of patients who had a preoperative blood sample characterized as $\mathrm{K}-R A S$ wild-type survived, whereas only approximately $15 \%$ of patients who had a preoperative blood sample characterized as $\mathrm{K}-R A S$ mutant survived. One study combined detection of $\mathrm{K}-R A S$ mutation with quantitative measurement of circulating plasma DNA [45]. They concluded that this approach could "confirm the presence of CRC, define disease-free status, and indicate the presence of relapse." Also, in a relatively small study examining pancreatic cancer patients treated with gefitinib, paclitaxel and radiation, patients demonstrating clearance of plasma K-RAS mutant DNA had longer survival times than patient with detectable $\mathrm{K}-R A S$ mutant plasma DNA following treatment [46].

The prognostic value of $\mathrm{K}-R A S$ mutational status with respect to NSCLC is much less clear. A meta-analysis of studies investigating this question found $\mathrm{K}-R A S$ mutation negatively impacts survival from NSCLC [47]. Gautschi et al. reported that survival of patients with mutant plasma $\mathrm{K}-R A S$ was significantly worse than that of patients with WT K-RAS [48]. By contrast, Camps et al. found no significant differences in NSCLC patient survival attributable to serum K-RAS mutation [49]. Part of the controversy regarding the use of $\mathrm{K}-R A S$ mutation as a prognostic biomarker for NSCLC or pancreatic cancer relates to finding different $\mathrm{K}-R A S$ mutations in circulating DNA and in tumor DNA [50,51].

\section{K-RAS mutation as a biomarker for targeted biological therapy selection}

Detection of mutation in the various molecular entities comprising the RAS-RAF-MEK-ERK kinase pathway, including $\mathrm{K}-R A S$ and EGFR, spurred development of therapies directed against these targets $[2,3]$. These included inhibitors of RAS farnesylation, antisense oligonucleotides against kinase signaling cascade molecules, small molecule kinase inhibitors directed against vascular endothelial growth 
factor, the extracellular domain of EGFR, or RAF isoforms (bevacizumab, erlotinib, gefitinib, sorafenib) and antibodies designed to block EGFR dimerization (cetuximab, panitumumab). A major turning point in the application of anti-EGFR therapies came when a number of studies observed that K-RAS mutational status was correlated with therapeutic response, specifically, a $\mathrm{K}-R A S$ mutant status could predict failure to respond to these therapies.

The importance of $\mathrm{K}-R A S$ mutational status was demonstrated in lung cancer patient response to EGFR-targeted therapy, where the response of lung adenocarcinoma patients to the EGFR thymidine kinase inhibitors, gefitinib and erlotinib, was stratified with respect to $\mathrm{K}-R A S$ mutation [52]. $\mathrm{K}-R A S$ mutation was detected in none of 12 tumors of patients sensitive to gefitinib therapy and in five out of 12 tumors of patients refractory to gefitinib therapy. K-RAS mutation was detected in none of the nine tumors of patients sensitive to erlotinib therapy and in four out of 26 tumors of patients refractory to erlotinib therapy. In a clinical trial examining the efficacy of erlotinib for advanced NSCLC, none of the five subjects showing a partial response had a $\mathrm{K}-R A S$ mutant tumor [53]. In a similar study, none of the 17 patients with a stage IIIB or IV $\mathrm{K}-R A S$ mutant NSCLC responded to erlotinib therapy [54]. In patients diagnosed with bronchioloalveolar carcinoma or adenocarcinoma (bronchioloalveolar carcinoma subtype), none of 18 patients with a $\mathrm{K}-R A S$ mutant tumor responded to erlotinib [55]. A meta-analysis of studies reporting responses of NSCLC patients to EGFR-targeted agents, as stratified by detection of $\mathrm{K}-R A S$ tumor mutation, concluded that $\mathrm{K}-R A S$ mutations are highly-specific negative predictors of response to single-agent EGFR thymidine kinase inhibitors [56].

The utility of cetuximab, a mouse-human chimeric monoclonal antibody directed against the EGFR, was investigated in patient populations with advanced CRC. In 2006, Lievre et al. stratified cetuximab responders and nonresponders by $\mathrm{K}-R A S$ mutational status [57]. Remarkably, they found that none of the 11 responders $(0 \%)$ had $\mathrm{K}-R A S$ mutant tumors, whereas 13 out of 19 (68.4\%) nonresponders had K-RAS mutant tumors. This was confirmed in a second study where none of the $12(0 \%)$ responders had K-RAS mutant tumors, but 17 out of $28(60.7 \%)$ patients whose disease progressed had a K-RAS mutant tumor [58]. De Roock et al. observed an objective response to cetuximab therapy in 27 out of 66 (40.9\%) metastatic colorectal cancer (mCRC) patients with WT K-RAS, whereas no response was seen in any $(0 \%)$ of the 42 patients with a $\mathrm{K}-R A S$ mutant tumor [59]. Frattini et al. observed a partial response to cetuximab therapy in nine out of $17(52.9 \%)$ mCRC patients with WT $\mathrm{K}-R A S$, but a partial response was observed in only one of ten $(10 \%)$ patients with a K-RAS mutant tumor [60]. Khambata-Ford et al. reported $\mathrm{K}-R A S$ mutation in tumors of three out of 27 subjects (11\%) who responded to cetuximab or had stable disease, whereas $\mathrm{K}-R A S$ mutation was detected in 27 out of 53 (51\%) tumors from nonresponders [61]. Compared with best supportive care alone in a randomized clinical trial, cetuximab treatment significantly improved overall survival and progression-free survival of advanced CRC patients with WT K-RAS tumors [62]. There was no significant difference in overall survival or progression-free survival in an otherwise similar patient population with K-RAS mutant tumors. Cetuximab's use as a first-line treatment, in combination with irinotecan, fluorouracil and leucovorin (FOLFIRI) has also been investigated and, again, the benefit from cetuximab therapy was limited to the K-RAS WT patient population [63].

Panitumumab, a fully-humanized monoclonal antibody directed against the EGFR, was used in several clinical trials for the treatment of patients with mCRC [2]. A meta-analysis of three Phase II clinical trials of panitumumab found $37 \%$ of WT patients had progressive disease compared with $79 \%$ of patients with a $\mathrm{K}-R A S$ mutant tumor [64]. In a Phase III clinical trial comparing panitumumab treatment to best supportive care for mCRC, Amado et al. observed the median progression-free survival with panitumumab treatment in the WT K-RAS group was 12.3 weeks, but only 7.3 weeks in the K-RAS mutant group [65]. Benvenuti et al. reported K-RAS mutation in one out of 11 responders (9\%) and 15 out of 37 nonresponders (40.5\%) to panitumumab or cetuximab therapies for CRCs [66].

Antibody-mediated EGFR blockade has become a major research focus and a number of review articles have described the clinical research in this area $[38,67,68]$. Several clinical trials investigating the efficacy of anti-EGFR antibody therapies in patients with advanced CRC are summarized in Taвze 2. Based on these and the additional studies mentioned above, the American Society of Clinical Oncology (ASCO) issued a provisional clinical opinion stating that these therapies should not be used in patients with K-RAS mutant tumors [69]. In addition, a label change for cetuximab, indicating that it has not been shown to be effective in colon 
Table 2. Impact of K-RAS mutational status on efficacy of antibody-mediated EGFR blockade in the treatment of advanced colorectal cancer.

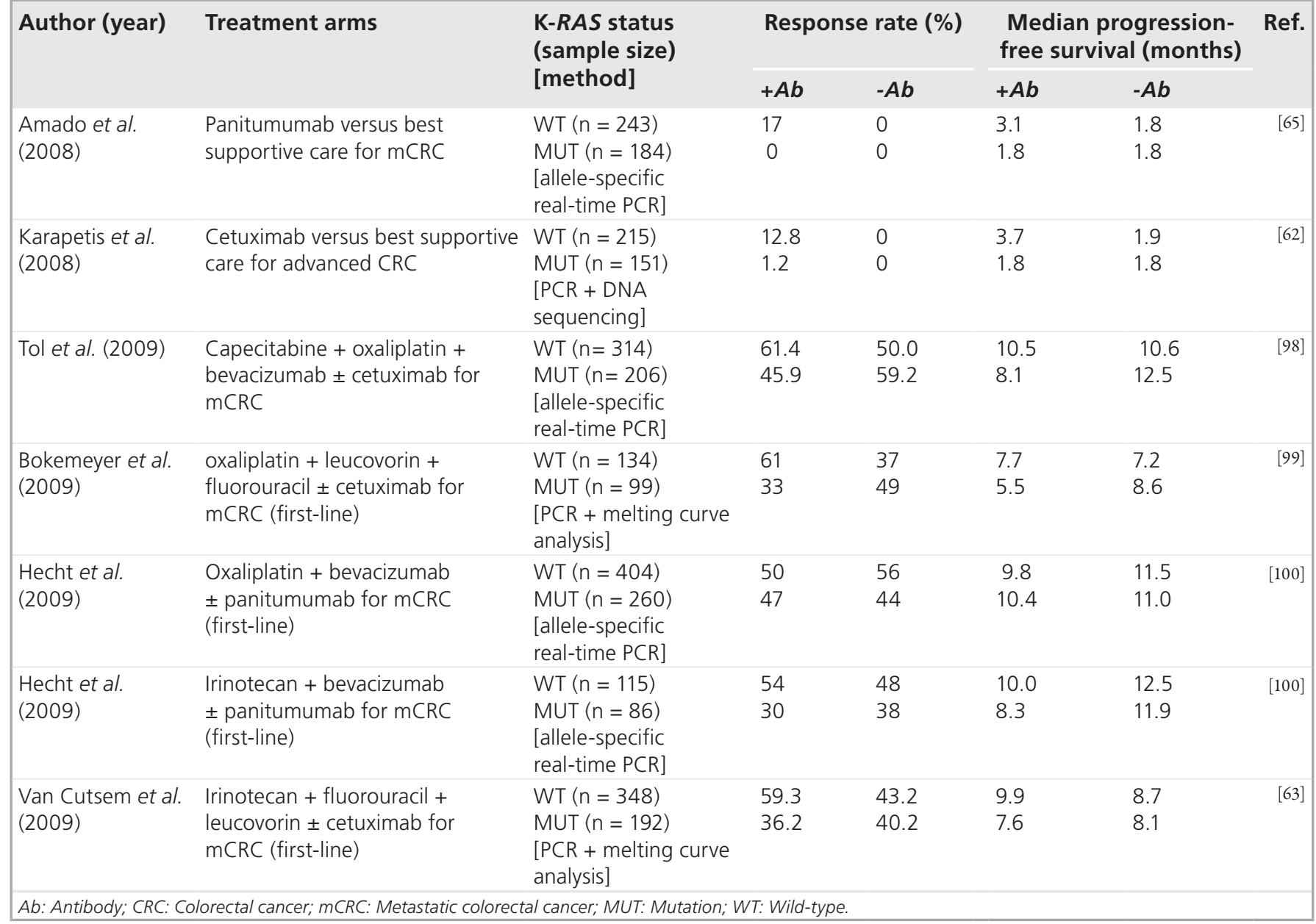

cancer patients with $\mathrm{K}-R A S$ mutant tumors, was recently approved by the US FDA. Clearly, these studies demonstrate the importance of characterizing tumors for $\mathrm{K}-R A S$ mutation as part of the routine management of cancer patients. A brief summary of the College of American Pathologists (CAP) Perspectives on Emerging Technology (POET) report is included in the ASCO provisional clinical opinion [69], whereas the complete report entitled 'KRAS mutation testing for colorectal cancer, February 2009 (Rev 2)' can be accessed from the CAP website [102]. By accurately characterizing patient tumors in terms of $\mathrm{K}-R A S$ mutation and applying the therapies described above only to patients with $\mathrm{K}-R A S \mathrm{WT}$ tumors, responses will be optimized and patients with $\mathrm{K}-R A S$ mutant tumors will not be burdened unnecessarily by ineffective and expensive therapies. Furthermore, given the evidence that different $\mathrm{K}-R A S$ mutations are associated with different clinical outcomes $[36,37,40]$, the specific K-RAS base substitution in a patient's tumor, rather than just the presence or absence of a $\mathrm{K}-R A S$ mutation, may need to be considered in the selection of therapeutic approach. Also, the fact that patients with $\mathrm{K}-R A S$ mutant tumors fail to respond to some of the targeted biological therapies highlights the need for effective therapies appropriate for use in patients with $K-R A S$ mutant tumors $[38,70]$.

Theoretically, therapies that specifically target RAS or signaling molecules downstream from RAS would be appropriate for the treatment of $\mathrm{K}-R A S$ mutant tumors. Considerable effort has been directed toward developing targeted biological therapies that abrogate the posttranslational processing of RAS, with the idea that mutant K-RAS will not function in signal transduction if it loses its membrane localization $[71,72]$. Vaccination of cancer patients with mutant K-RAS peptide was performed with the goal of developing tumor-specific immunity [73]. The use of small interfering RNA molecules to knock-down mutant $\mathrm{K}-R A S$ expression is being investigated [74]. Nevertheless, there is a need for additional research in this area. 


\section{Nature of tumor-associated mutations, methodologies for mutation characterization \& data interpretation}

Now that K-RAS mutational status has demonstrable clinical significance in the care of cancer patients, understanding the complexities involved in characterizing $\mathrm{K}-R A S$ mutational status and the strengths and weaknesses of the methodologies used to characterize $\mathrm{K}-R A S$ mutational status has become quite important. Some issues that should be considered are:

- The need to detect tumor-associated mutations, such as $\mathrm{K}-R A S$, as subpopulations within a tumor tissue sample;

- The tissue sampling approach most appropriate for detecting subpopulations of tumor cells within a patient's tissue sample;

- The sensitivity required;

- The feasibility of achieving that sensitivity in the context of patient care;

- Determining the best metric with which to describe $\mathrm{K}-R A S$ mutation.

Monoclonal tumor origin persists as a default assumption and significantly influences interpretation of tumor mutation research. Over the years considerable evidence has been collected and published in a number of review articles indicating that many, perhaps most, tumors are polyclonal in origin [75-77]. The clearest example is colon tumors, for which there is definitive evidence of polyclonal tumor origin. Polyclonal tumor origin complicates expectations regarding how $\mathrm{K}-R A S$ mutations will persist during tumor development. Most importantly, polyclonal tumor origin predicts $\mathrm{K}-R A S$ mutations will be present as subpopulations in many or most tumors, and using sensitive and quantitative methodologies, this has proven to be the case [31,78,79]. Dieterle et al. reported ratios of K-RAS mutant:WT DNA ranged over four orders of magnitude $\left(10^{0}-10^{-4}\right)$, with the median at approximately $10^{-2}[78]$. Additional indirect evidence that tumors contain $\mathrm{K}-R A S$ mutant subpopulations comes from studies that compare a sensitive mutation detection approach to standard DNA sequencing and report higher numbers of $\mathrm{K}-R A S$-positive tumors using the more sensitive methodology [80,81]. For example, Beau-Faller $e t$ al. detected a subset of $\mathrm{K}-R A S$-positive tumors using a peptide nucleic acid-mediated PCR clamping technique that were $\mathrm{K}-R A S$ negative by DNA sequencing, even though the samples were characterized as having a sufficient percentage of tumor cells for DNA sequencing analysis [79]. They concluded these samples had mutant tumor subclones.

Mutant subpopulations of tumor cells can be explained in two different ways, an initial heterogeneity due to polyclonal tumor origin, or heterogeneity that arises during tumor progression. Even when K-RAS is an initiating mutation, additional heterogeneity may occur during polyclonal tumor outgrowth. For example, a $\mathrm{K}-R A S$ mutant clone might be outgrown by a more aggressive, initially complementing clone carrying a tumor suppressor gene mutation. There is considerable evidence in the literature of initial heterogeneity in preneoplastic lesions that is lost in the carcinoma stage, suggesting a single clone frequently predominates as tumors progress and metastasize [77]. Indeed, it has been concluded that "considering the genetic heterogeneity of colorectal cancers, the absence of detectable $K-R A S$ mutations in the primary tumor cannot formally exclude the presence of KRAS mutation in metastases" [82]. There is evidence that $\mathrm{K}-R A S$ codon 12 GTT mutations are lost during colonic adenoma to adenocarcinoma progression [31]. This quantitative data is consistent with the qualitative colon tumor data in the COSMIC database, where 36 out of 352 adenomas (10.2\%) but only 146 out of 1976 adenocarcinomas $(7.4 \%)$ carry the $\mathrm{K}-R A S$ codon 12 GTT mutation [101]. Conversely, K-RAS codon 12 GAT mutation becomes more prevalent during progression; 39 out of 352 adenomas (11.1\%) and 262 out of 1976 adenocarcinomas (13.3\%) carry the $\mathrm{K}-R A S$ codon 12 GAT mutation [101]. This aspect of plasticity in tumor-associated mutations may explain why numerous studies report a fraction of serum, plasma, or urine samples contain a different $\mathrm{K}-R A S$ mutation than that found in the patient's tumor $[29,83,84]$. Regardless of their origin or plasticity during tumor progression, the fact that $\mathrm{K}-R A S$ mutations are present as subpopulations in tumors means that methodologies more sensitive than standard DNA sequencing are needed to accurately interpret their significance.

The impact of tumor subpopulations on the results of clinical trials is an open question. It is clear that patients with $\mathrm{K}-R A S$ mutant tumors fail to respond to therapies directed against the EGFR. What is unclear, however, is to what extent patients characterized as K-RAS WT have undetected $\mathrm{K}-R A S$ mutant tumor subpopulations that affect their response to therapy. In this regard, it is important to note that clinical trials find significant fractions of K-RAS WT patients who also do not respond to anti-EGFR therapies. 
Failure to respond may be due to a genetic lesion in a different molecular entity in the RAFMEK-ERK kinase pathway. However, until $\mathrm{K}-R A S$ mutant:WT levels are determined and associated with particular therapeutic responses, the possibility that failure of anti-EGFR therapies is due to undetected $\mathrm{K}-R A S$ mutant tumor subpopulations cannot be excluded. One study, however, suggests that small $\mathrm{K}-R A S$ subpopulations may not be clinically important [79]. A weak association was detected between poor event-free survival of NSCLC patients and K-RAS mutation (as measured by DNA sequencing); however, the association was not apparent using mutational data collected with a more sensitive method.

Because $\mathrm{K}-R A S$ mutation has become important to the practice of oncology, clinicians will need to work closely with pathologists to select a molecular diagnostic approach and appropriate samples to analyze [85]. K-RAS mutation testing may be performed in house at many oncology centers. Although there is currently no US FDAapproved test for $\mathrm{K}-R A S$ mutation, several companies currently provide $\mathrm{K}-R A S$ testing services and their numbers are likely to increase in the future $[69,85]$. A variety of methods have been developed for the analysis of $\mathrm{K}-R A S$ mutation and have been reviewed [85-87]. Reliable approaches for K-RAS mutation detection include methods based on separation of electrophorectic variant sequence, including single-strand conformation polymorphism (SSCP), heteroduplex analysis (HA), thermal gradient capillary electrophoresis (TGCE) and constant denaturation capillary electrophoresis. TaвLe 3 describes a sampling of assays and technologies recently used to characterize the K-RAS mutation in clinical specimens. TAвLE 3 also illustrates that assay sensitivity may not be established in the context of clinical sample analyses and that most of the approaches for the analysis of clinical samples are not quantitative. As additional personalized oncology therapies are developed, the use of companion diagnostics may be evaluated as part of clinical trials, thereby providing efficacy information for a drug in the context of its use with a companion diagnostic $[88,103]$. It is likely that the clinical need for personalized patient information will drive development of more sensitive and quantitative methods to characterize tumor-associated mutations. Because of the need to detect mutant subpopulations of tumor cells, sensitivity is a critical issue in companion diagnostic development. A recent review describes a proposal for a European quality assurance program as it relates to clinical K-RAS mutation testing [86]. The recommendation put forward in this review is that "a lower detection limit of mutant signal should be set at $1 \%$ of tumor cells for allelespecific PCR and 25-30\% for direct sequencing." However, as mentioned above, when a sensitive methodology was applied, colon tumors were shown to have a median $\mathrm{K}-R A S$ mutant fraction of approximately $10^{-2}$ [78]. Therefore, a cut-off of $1 \%$ would fail to detect $\mathrm{K}-R A S$ mutations in approximately half of all samples, and a majority of $\mathrm{K}-R A S$ mutations would be missed if samples were analyzed by standard DNA sequencing. Presumably, the $1 \%$ cut-off is based on the idea that low levels of $\mathrm{K}-R A S$ mutation will not be clinically relevant. However, the clinical significance of low levels of K-RAS mutation cannot be known until they are quantified and particular levels of mutation are associated with clinical response. The possibility that therapies directed against predominantly WT K-RAS tumors could provide an opportunity for the clonal outgrowth of small subpopulations of $\mathrm{K}-R A S$ mutant tumor cells should be considered.

Several aspects of clinical sample analysis require additional investigation and optimization. Methods for circulating tumor DNA isolation from plasma or urine samples need to be optimized because different DNA isolation approaches have been shown to produce different results $[84,89]$. Optimization of sample collection techniques also needs to be considered. The proposal for the European quality assurance program suggests that the optimal material is to evaluate for $\mathrm{K}-R A S$ mutation are preserved tissue blocks from the primary tumor, if available, or its metastases [86]. If $\mathrm{K}-R A S$ mutational testing is to become a routine part of clinical care, when appropriate, the storage of a representative portion of a patient's tumor as unfixed frozen tissue is much preferable for sensitive mutational analyses. If $\mathrm{K}-R A S$ mutation is present as small subpopulations within tumors, then complete exclusion of nontumor cells becomes less important than analyzing a fairly large representative sample of tumor tissue and avoiding artifacts due to fixation. Methodologies used to characterize $\mathrm{K}-R A S$ mutation need to be quantitative. The use of any end point as a cancer biomarker in the absence of quantification is unlikely to produce consistently useful results. While sensitive and quantitative research methods to measure $\mathrm{K}-R A S$ mutation exist, many are too cumbersome to be appropriate in a clinical setting $[78,90]$. Fortunately, there are a few technologies on the horizon that may prove useful in this regard. One promising approach described by Diehl et al. uses real-time PCR to quantify the amount of circulating tumor DNA 


\section{Table 3. Recent approaches to K-RAS mutational analysis*.}

\section{Method name}

RFLP-PCR

\section{Method description}

Measures mutations that alter a restriction endonuclease recognition sequence; digestion by a restriction enzyme reduces the amount of wild-type sequence, PCR with primers flanking the restriction site amplifies the uncut mutant sequence

Mutant allele-specific amplification with denaturing high-pressure liquid chromatography (MASA/ DHPLC)

Peptide nucleic acid-mediated PCR clamping and melting curve analysis

PCR, mutant allele enrichment, and denaturing gradient gel electrophoresis (PCR/MAE/DGGE) Isothermal multiple displacement amplification (IMDA)

Membrane array

Real-Time PCR and DNA melting curve analysis

Capillary PCR with peptide nucleic acid clamp/sensor probe

Enriched-PCR/RFLP

Real-time PCR with peptide nucleic acid clamp

COLD-PCR/sequencing

Single-strand conformation polymorphism-capillary electrophoresis (SSCP-CE)

Mutant allele-specific amplification with electrochemiluminescence (MASA/ECL)

An allele-specific PCR ( 3 ' end of primer matches mutation being detected) is followed by heteroduplex formation and identification of heteroduplexes by DHPLC

Capillary PCR performed using a wild-type peptide nucleic acid clamp and melting temperature analysis performed using mutant-specific probes with different fluorophores

PCR amplification followed by restriction digestion to enrich for mutant sequence, then analyzed by DGGE

IMDA provides uniform amplification of clinical sample

DNA, K-RAS mutation was analyzed by PCR and DNA sequencing

Poly $(A)+$ RNA isolated from blood is used to synthesize a CDNA and probe membranes containing 22 genes up-regulated by mutant K-RAS

Real-time PCR performed on a LightCycler with specific anchor probes. Melting of PCR product and anchor probe is monitored as fluorescence, with specific K-RAS mutations identified by Tm

Capillary PCR performed with a peptide nucleic acid complementary to the wild-type sequence and spanning codons 12 and 13 of $\mathrm{K}$-RAS reduces wild-type amplification and acts as sensor probe in melting temperature analysis Multiple rounds of PCR and digestion of WT K-RAS codon 12 , followed by direct sequencing

Real-time PCR performed in the presence of a peptide nucleic acid sequence specific for wild-type K-RAS codons 12 and 13

Preferentially enriches 'minority alleles' by converting mutant into heteroduplex and performing PCR using a temperature that will denature heteroduplex but not abundant homoduplex, mutations are identified by DNA sequencing

Real-time PCR with bead, emulsion, Sequence of interest is amplified in a PCR adding tag amplification and magnetics (BEAMing) sequences, the tag sequences are used to amplify and attached DNA to beads isolated in emulsions, specific sequences are identified by fluorophore-specific oligonucleotide hybridization analyzed by flow cytometry

Two oligonucleotides are hybridized adjacent to the basepair being interrogated, if a base substitution is present in the target DNA the oligonucleotides are ligated and then detected by real-time, quantitative PCR

Combined a universal fluorescent primer with PCR products mutant in codons 12 or 13 separated and identified by $C E$

An allele-specific PCR ( $3^{\prime}$ end of primer matches mutation being detected) conducted on magnetic beads with detection based on ECL emission of Ru(bpy) ${ }_{3}^{2+}$

\section{Sensitivity Quantitative \\ Ref.}

$10^{-6}-10^{-8 \ddagger} \quad$ No

[11]

$10^{-3 \neq}$

No

$[8]$

$\sim 10^{-3}-10^{-4} \quad$ No

[92]

$\sim 10^{-4}-10^{-5 \neq} \quad$ No

[19]

NSR

No

[93]

5 mutant

No

cells $/ 1 \mathrm{ml}$ of

blood

$10^{-3}$

No

[81]

$10^{-4}$

No

[13]

NSR

No

[14]

NSR

No

[82]

$10^{-2}$

No

[95]

$10^{-4}$

Yes

$[10,91]$

"Quantification is rare and studies do not always document the sensitivity of applied methods.

"Sensitivity cited by authors, but not demonstrated/evaluated as part of the study. CE: Capillary electrophoresis; COLD: Co-amplification at lower denaturation temperature; DHPLC: Denaturing high-pressure liquid chromatography; ECL: Electrochemiluminescence; NSR: No sensitivity reported; RFLP: Restriction fragment length polymorphism. 
in plasma, along with an approach called 'beaming' (TABLE 3 ) to determine the ratio of mutant:WT DNA in the circulating tumor DNA [10,91]. The 'LigAmp' assay is another promising approach that has been used successfully to establish clinically relevant levels of $\mathrm{K}-R A S$ mutation [34].

\section{Conclusion}

Detection of $\mathrm{K}-R A S$ mutation in various bodily fluids cannot be equated with cancer and is unlikely to lead to a successful cancer screening strategy. Approaches that quantify K-RAS mutation in plasma or urine and use a level of $\mathrm{K}-R A S$ mutation as a trigger for additional cancer screening have potential and should be investigated. The presence of $\mathrm{K}-R A S$ mutation in a patient's tumor does have clinical significance in terms of patient prognosis and therapy selection.

\section{Future perspective}

As the archetype mutational biomarker, past experiences with $\mathrm{K}-R A S$ mutation in the clinical setting should inform future efforts toward personalized medicine. K-RAS mutation may be present in a subpopulation of tumor cells and $\mathrm{K}-R A S$ mutant fraction may change during progression and metastasis, making the measurement of $\mathrm{K}-R A S$ more challenging than is generally recognized. Determining if $\mathrm{K}-R A S$ mutation is present or absent from patient samples is not a sufficiently rigorous characterization of mutational status. Progress in understanding the significance of particular levels of $\mathrm{K}-R A S$ mutations with respect to therapeutic outcomes will only be achieved when quantitative data begin to be collected and analyzed.

\section{Acknowledgements}

The authors thank Robert Heflich and Page McKinzie for their critical review of the manuscript.

\section{Financial \& competing interests disclosure}

The opinions and information in this review article are those of the authors, and do not represent the views and/or policies of the US Food and Drug Administration.

The authors have no relevant affiliations or financial involvement with any organization or entity with a financial interest in or financial conflict with the subject matter or materials discussed in the manuscript. This includes employment, consultancies, honoraria, stock ownership or options, expert testimony, grants or patents received or pending, or royalties.

No writing assistance was utilized in the production of this manuscript.

\section{Executive summary}

\section{Significance of $K$-RAS as a mutational biomarker}

- K-RAS mutations are detected in significant fractions of many human cancers.

- K-RAS mutation disrupts normal cell proliferation and induces prosurvival factors, thus the K-RAS mutant phenotype drives carcinogenesis.

- Mutations are clustered at codon 12, making K-RAS codon 12 the most cancer-prevalent mutational target.

\section{$K$-RAS mutation in cancer screening}

- Studies have investigated K-RAS mutation in blood/plasma/serum, urine, stool, pancreatic juice, sputum and bronchoalveolar lavage fluid.

- To date, no cost-effective cancer screening strategy has been developed based on K-RAS mutation.

\section{$K$-RAS mutation in cancer prognosis}

- In colorectal cancer patients, K-RAS mutation, whether measured in tumor or plasma/serum, has been associated with poor prognosis.

- In non-small-cell lung cancer patients, the value of plasma/serum K-RAS as a prognostic biomarker remains an open question.

\section{$K$-RAS mutation in personalized medicine}

- K-RAS mutation predicts failure to respond to targeted biological therapies directed against the EGFR.

- Better approaches for treating patients with K-RAS mutant tumors are needed.

\section{Nature of tumor-associated mutations \& methods for mutation characterization}

- K-RAS mutation can be present in subpopulations of tumor cells.

- Sensitive mutation detection methods frequently detect mutations in tumors that are missed by standard DNA sequencing.

- For personalized use of targeted biological therapies to be successful, sensitive and quantitative measurement of tumor-associated mutations will be required.

\section{Bibliography}

Papers of special note have been highlighted as:

- of interest

"w of considerable interest

1 Moon A: Differential functions of ras for malignant phenotypic conversion. Arch. Pharm. Res. 29, 113-122 (2006).
2 Ciardiello F, Tortora G: EGFR antagonists in cancer treatment. N. Engl. J. Med. 358, 1160-1174 (2008).

3 Downward J: Targeting RAS signalling pathways in cancer therapy. Nat. Rev. Cancer 3, 11-22 (2003).

- Includes an excellent summary of signaling through EGFR, plus a history of anti-EGFR therapies and a description of their clinical applications.

4 Regina S, Rollin J, Blechet C, Iochmann S, Reverdiau P, Gruel Y: Tissue factor expression in non-small cell lung cancer: relationship with vascular endothelial growth factor expression, microvascular density, and K-ras mutation. J. Thorac. Oncol. 3, 689-697 (2008). 
5 Cespedes MV, Sancho FJ, Guerrero $S$ et al:: K-ras Asp12 mutant neither interacts with Raf, nor signals through Erk and is less tumorigenic than K-ras Val12. Carcinogenesis 27, 21902200 (2006).

6 Janssen KP, Abal M, El Marjou F, Louvard D, Robine S: Mouse models of K-ras-initiated carcinogenesis. Biochim. Biophys. Acta 1756, 145-154 (2005).

7 Forbes SA, Bhamra G, Bamford S et al.: The catalogue of somatic mutations in cancer (COSMIC). Current Protocols in Human Genetics 57 (UNIT 10.11), 11-26 (2008).

8 Lilleberg SL, Durocher J, Sanders C, Walters K, Culver K: High sensitivity scanning of colorectal tumors and matched plasma DNA for mutations in $A P C, T P 53, K-R A S$, and $B R A F$ genes with a novel DHPLC fluorescence detection platform. Ann. NY Acad. Sci. 1022, 250-256 (2004)

9 Su YH, Wang M, Aiamkitsumrit B, Brenner DE, Block TM: Detection of a K-ras mutation in urine of patients with colorectal cancer. Cancer Biomark. 1, 177-182 (2005).

10 Diehl F, Schmidt K, Durkee KH et al.: Analysis of mutations in DNA isolated from plasma and stool of colorectal cancer patients. Gastroenterology 135, 489-498 (2008).

- Describes a sensitive, quantitative approach for mutant status characterization, with the potential for broad application in clinical sample analysis.

11 Kimura T, Holland WS, Kawaguchi T et al.: Mutant DNA in plasma of lung cancer patients: potential for monitoring response to therapy. Ann. NY Acad. Sci. 1022, 55-60 (2004).

12 Bremnes RM, Sirera R, Camps C: Circulating tumour-derived DNA and RNA markers in blood: a tool for early detection, diagnostics, and follow-up? Lung Cancer 49, 1-12 (2005).

13 Luo JD, Chan EC, Shih CL et al:: Detection of rare mutant K-ras DNA in a single-tube reaction using peptide nucleic acid as both PCR clamp and sensor probe. Nucleic Acids Res. 34, E12 (2006).

14 Jiao L, Zhu J, Hassan MM, Evans DB, Abbruzzese JL, Li D: K-ras mutation and p16 and preproenkephalin promoter hypermethylation in plasma DNA of pancreatic cancer patients: In relation to cigarette smoking. Pancreas 34, 55-62 (2007).

15 Su YH, Wang M, Block TM et al.: Transrenal DNA as a diagnostic tool: Important technical notes. Ann. NY Acad. Sci. 1022, 81-89 (2004).

16 Lichtenstein AV, Melkonyan HS, Tomei LD, Umansky SR: Novel applications of polymerase chain reaction to urinary nucleic acid analysis. Methods Mol. Biol. 336, 145-154 (2006).
17 Su YH, Wang M, Brenner DE et al.: Human urine contains small, 150 to 250 nucleotidesized, soluble DNA derived from the circulation and may be useful in the detection of colorectal cancer. J. Mol. Diagn. 6, 101-107 (2004).

18 Destro A, Bianchi P, Alloisio M et al.: K-ras and p16(INK4A)alterations in sputum of NSCLC patients and in heavy asymptomatic chronic smokers. Lung Cancer 44, 23-32 (2004).

19 Keohavong P, Gao WM, Zheng KC et al: : Detection of K-ras and p 53 mutations in sputum samples of lung cancer patients using laser capture microdissection microscope and mutation analysis. Anal. Biochem. 324, 92-99 (2004).

20 Gormally E, Vineis P, Matullo G et al.: TP53 and KRAS2 mutations in plasma DNA of healthy subjects and subsequent cancer occurrence: a prospective study. Cancer Res. 66, 6871-6876 (2006).

21 Khalid A, Finkelstein S, Mcgrath K: Molecular diagnosis of solid and cystic lesions of the pancreas. Clin. Lab. Med. 25, 101-116 (2005).

22 Loktionov A: Cell exfoliation in the human colon: myth, reality and implications for colorectal cancer screening. Int. J. Cancer 120, 2281-2289 (2007).

23 Imperiale TF, Ransohoff DF, Itzkowitz SH, Turnbull BA, Ross ME: Fecal DNA versus fecal occult blood for colorectal-cancer screening in an average-risk population. N. Engl. J. Med. 351, 2704-2714 (2004).

24 Ouyang DL, Chen JJ, Getzenberg RH, Schoen RE: Noninvasive testing for colorectal cancer: a review. Am. J. Gastroenterol. 100, 1393-1403 (2005).

25 Loktionov A: Common gene polymorphisms, cancer progression and prognosis. Cancer Lett. 208, 1-33 (2004).

26 Rennert G, Kislitsin D, Brenner DE, Rennert HS, Lev Z: Detecting K-ras mutations in stool from fecal occult blood test cards in multiphasic screening for colorectal cancer. Cancer Lett. 253, 258-264 (2007).

27 Whitney D, Skoletsky J, Moore K et al.: Enhanced retrieval of DNA from human fecal samples results in improved performance of colorectal cancer screening test. J. Mol. Diagn. 6, 386-395 (2004).

28 Olson J, Whitney DH, Durkee K, Shuber AP: DNA stabilization is critical for maximizing performance of fecal DNA-based colorectal cancer tests. Diagn. Mol. Pathol. 14, 183-191 (2005).

29 Neri M, Cesario A, Granone P et al.: Prognostic role of K-Ras mutations in non-small cell lung cancer: still an issue for open debate. Lung Cancer 53, 393-395; author reply 397-398 (2006).
30 Yamada S, Yashiro M, Maeda K, Nishiguchi Y, Hirakawa K: A novel high-specificity approach for colorectal neoplasia: detection of K-ras 2 oncogene mutation in normal mucosa. Int. J. Cancer. 113, 1015-1021 (2005).

31 Parsons BL, Marchant KE, Verkler TL et al: Quantification of K-RAS codon 12 GAT and GTT mutation in colon tumor and non-tumor tissue by allele-specific competetive blocker PCR. Presented at: The Centennial Annual Meeting of the American Association for Cancer Research. Los Angeles, CA, USA, 14-18 April 2007 (abstract 5245).

32 Magistrelli P, Neri M, Granone P, Cesario A, Paleari L, Russo P: K-ras mutations in circulating DNA from pancreatic and lung cancers: bridging methodology for a common validation of the molecular diagnosis value. Pancreas 37, 101-102 (2008).

33 Sawabu N, Watanabe H, Yamaguchi Y, Ohtsubo K, Motoo Y: Serum tumor markers and molecular biological diagnosis in pancreatic cancer. Pancreas 28, 263-267 (2004).

34 Shi C, Fukushima N, Abe T et al:: Sensitive and quantitative detection of KRAS2 gene mutations in pancreatic duct juice differentiates patients with pancreatic cancer from chronic pancreatitis, potential for early detection. Cancer Biology Therapy 7, 353-360 (2008).

35 Osborn NK, Ahlquist DA: Stool screening for colorectal cancer: molecular approaches. Gastroenterology 128, 192-206 (2005).

36 Andreyev HJ, Norman AR, Cunningham D, Oates JR, Clarke PA: Kirsten ras mutations in patients with colorectal cancer: the multicenter "RASCAL" Study. J. Natl. Cancer Inst. 90, 675-684 (1998).

37 Andreyev HJ, Norman AR, Cunningham D et al.: Kirsten ras mutations in patients with colorectal cancer: The 'RASCAL II' study. Br. J. Cancer 85, 692-696 (2001).

38 Jiang Y, Kimchi ET, Staveley-O'Carroll KF, Cheng H, Ajani JA: Assessment of K-ras mutation, a step toward personalized medicine for patients with colorectal cancer. Cancer 115, 3609-3617 (2009).

39 Chang YS, Yeh KT, Chang TJ et al.: Fast simultaneous detection of $K-R A S$ mutations in colorectal cancer. BMC Cancer 9, 179 (2009).

40 Winder T, Scheithauer W, Lang A: K-ras mutations and cetuximab in colorectal cancer. N. Engl. J. Med. 360, 834-835; author reply 835-836 (2009).

41 Riely GJ, Kris MG, Rosenbaum D et al.: Frequency and distinctive spectrum of $K R A S$ mutations in never smokers with lung adenocarcinoma. Clin. Cancer Res. 14, 5731-5734 (2008). 
42 Wang JY, Hsieh JS, Chang MY et al.: Molecular detection of $A P C, \mathrm{~K}-r a s$, and $p 53$ mutations in the serum of colorectal cancer patients as circulating biomarkers. World J. Surg. 28, 721-726 (2004).

43 Trevisiol C, Di Fabio F, Nascimbeni R et al.: Prognostic value of circulating KRAS2 gene mutations in colorectal cancer with distant metastases. Int. J. Biol. Markers 21, 223-228 (2006).

44 Bazan V, Bruno L, Augello C et al.: Molecular detection of TP53, Ki-Ras and p16 $6^{\mathrm{INK} 4 \mathrm{~A}}$ promoter methylation in plasma of patients with colorectal cancer and its association with prognosis. Results of a 3-year GOIM (Gruppo Oncologico dell'Italia Meridionale) prospective study. Ann. Oncol. 17(Suppl. 7), vii84-vii90 (2006).

45 Frattini M, Gallino G, Signoroni $S$ et al.: Quantitative and qualitative characterization of plasma DNA identifies primary and recurrent colorectal cancer. Cancer Lett. 263, 170-181 (2008).

46 Olsen CC, Schefter TE, Chen $\mathrm{H}$ et al.: Results of Phase I trial of 12 patients with locally advanced pancreatic carcinoma combining gefitinib, paclitaxel, and 3-dimensional conformal radiation. Am. J. Clin. Oncol. 32, 115-121 (2009).

47 Mascaux C, Iannino N, Martin B et al.: The role of RAS oncogene in survival of patients with lung cancer: a systematic review of the literature with meta-analysis. Br. J. Cancer 92, 131-139 (2005).

48 Gautschi O, Huegli B, Ziegler A et al:: Origin and prognostic value of circulating KRAS mutations in lung cancer patients. Cancer Lett. 254, 265-273 (2007).

49 Camps C, Sirera R, Bremnes R et al:: Is there a prognostic role of $\mathrm{K}$-ras point mutations in the serum of patients with advanced non-small cell lung cancer? Lung Cancer 50, 339-346 (2005).

50 Neri M, Cesario A, Granone P et al:: Prognostic role of $K$-ras mutations in non-small cell lung cancer: still an issue for open debate. Lung Cancer 53, 393-395 (2006).

51 Marchese R, Muleti A, Pasqualetti P et al.: Low correspondence between K-ras mutations in pancreatic cancer tissue and detection of K-ras mutations in circulating DNA. Pancreas 32, 171-177 (2006).

52 Pao W, Wang TY, Riely GJ et al.: KRAS mutations and primary resistance of lung adenocarcinomas to gefitinib or erlotinib. PLoS Med. 2, E17 (2005).

53 Jackman DM, Yeap BY, Lindeman NI et al.: Phase II clinical trial of chemotherapy-naive patients $>$ or $=70$ years of age treated with erlotinib for advanced non-small-cell lung cancer. J. Clin. Oncol. 25, 760-766 (2007).
54 Schneider CP, Heigener D, Schott-VonRomer $\mathrm{K}$ et al: : Epidermal growth factor receptor-related tumor markers and clinical outcomes with erlotinib in non-small cell lung cancer: an analysis of patients from German centers in the TRUST study. J. Thoracic Oncol. 3, 1446-1453 (2008).

55 Miller VA, Riely GJ, Zakowski MF et al.: Molecular characteristics of bronchioloalveolar carcinoma and adenocarcinoma, bronchioloalveolar carcinoma subtype, predict response to erlotinib. J. Clin. Oncol. 26, 1472-1478 (2008).

56 Linardou H, Dahabreh IJ, Kanaloupiti D et al:: Assessment of somatic K-RAS mutations as a mechanism associated with resistance to EGFR-targeted agents: a systematic review and meta-analysis of studies in advanced non-small-cell lung cancer and metastatic colorectal cancer. Lancet Oncol. 9, 962-972 (2008).

57 Lievre A, Bachet JB, Le Corre D et al.: KRAS mutation status is predictive of response to cetuximab therapy in colorectal cancer. Cancer Res. 66, 3992-3995 (2006).

"- Important in establishing that K-RAS mutant tumor status could predict failure to respond to therapies directed against the EGFR.

58 Di Fiore F, Blanchard F, Charbonnier F et al: : Clinical relevance of $K R A S$ mutation detection in metastatic colorectal cancer treated by cetuximab plus chemotherapy. $B r$. J. Cancer 96, 1166-1169 (2007).

59 De Roock W, Piessevaux H, De Schutter J et al: KRAS wild-type state predicts survival and is associated to early radiological response in metastatic colorectal cancer treated with cetuximab. Ann. Oncol. 19, 508-515 (2008).

60 Frattini M, Saletti P, Romagnani E et al.: PTEN loss of expression predicts cetuximab efficacy in metastatic colorectal cancer patients. Br. J. Cancer 97, 1139-1145 (2007).

61 Khambata-Ford S, Garrett CR, Meropol NJ et al:: Expression of epiregulin and amphiregulin and $K$-ras mutation status predict disease control in metastatic colorectal cancer patients treated with cetuximab. J. Clin. Oncol. 25, 3230-3237 (2007).

62 Karapetis CS, Khambata-Ford S, Jonker DJ et al.: K-ras mutations and benefit from cetuximab in advanced colorectal cancer. N. Engl. J. Med. 359, 1757-1765 (2008).

63 Van Cutsem E, Kohne CH, Hitre E et al: Cetuximab and chemotherapy as initial treatment for metastatic colorectal cancer. N. Engl. J. Med. 360, 1408-1417 (2009).

64 Freeman DJ, Juan T, Reiner M et al.: Association of K-ras mutational status and clinical outcomes in patients with metastatic colorectal cancer receiving panitumumab alone. Clin. Colorectal Cancer 7, 184-190 (2008).

65 Amado RG, Wolf M, Peeters M et al:: Wild-type $K R A S$ is required for panitumumab efficacy in patients with metastatic colorectal cancer. J. Clin. Oncol. 26, 1626-1634 (2008).

66 Benvenuti S, Sartore-Bianchi A, Di Nicolantonio F et al: : Oncogenic activation of the RAS/RAF signaling pathway impairs the response of metastatic colorectal cancers to anti-epidermal growth factor receptor antibody therapies. Cancer Res. 67, 2643-2648 (2007).

67 Staal S, O'connell MJ, Allegra CJ: The marriage of growth factor inhibitors and chemotherapy: bliss or bust? J. Clin. Oncol. 27, 1545-1548 (2009).

68 Chau I, Cunningham D: Treatment in advanced colorectal cancer: what, when and how? Br. J. Cancer 100, 1704-1719 (2009).

69 Allegra CJ, Jessup JM, Somerfield MR et al.: American Society of Clinical Oncology Provisional Clinical Opinion: testing for $K R A S$ gene mutations in patients with metastatic colorectal carcinoma to predict response to anti-epidermal growth factor receptor monoclonal antibody therapy. J. Clin. Oncol. 27, 2091-2096 (2009).

70 Riely GJ, Marks J, Pao W: KRAS mutations in non-small cell lung cancer. Proc. Am. Thorac. Soc. 6, 201-205 (2009).

71 Appels NM, Beijnen JH, Schellens JH: Development of farnesyl transferase inhibitors: a review. Oncologist 10, 565-578 (2005).

72 Morgan MA, Ganser A, Reuter CW: Targeting the RAS signaling pathway in malignant hematologic diseases. Curr. Drug Targets 8, 217-235 (2007).

73 Carbone DP, Ciernik IF, Kelley MJ et al.: Immunization with mutant $p 53$ - and $K$-ras-derived peptides in cancer patients: Immune response and clinical outcome. J. Clin. Oncol. 23, 5099-5107 (2005).

74 Brunner TB, Cengel KA, Hahn SM et al.: Pancreatic cancer cell radiation survival and prenyltransferase inhibition: the role of K-Ras. Cancer Res. 65, 8433-8441 (2005).

75 Duggan BJ, Gray SB, Mcknight JJ, Watson CJ, Johnston SR, Williamson KE:

Oligoclonality in bladder cancer: the implication for molecular therapies. J. Urol. 171, 419-425 (2004).

76 Halberg RB, Dove WF: Polyclonal tumors in the mammalian intestine: are interactions among multiple initiated clones necessary for tumor initiation, growth, and progression? Cell Cycle 6, 44-51 (2007). 
77 Parsons BL: Many different tumor types have polyclonal tumor origin: evidence and implications. Mutat. Res. 659, 232-247 (2008).

-1. A discussion of how X chromosome inactivation data has been misinterpreted and a review of evidence supporting polyclonal tumor origin.

78 Dieterle CP, Conzelmann M, Linnemann U, Berger MR: Detection of isolated tumor cells by polymerase chain reaction-restriction fragment length polymorphism for K-ras mutations in tissue samples of 199 colorectal cancer patients. Clin. Cancer Res. 10, 641-650 (2004).

-1" Establishes that K-RAS mutation is present in colon tumors as subpopulations.

79 Beau-Faller M, Legrain M, Voegeli AC et al.: Detection of $K$-Ras mutations in tumour samples of patients with non-small cell lung cancer using PNA-mediated PCR clamping. Br. J. Cancer 100, 985-992 (2009).

80 Marchetti A, Martella C, Felicioni L et al.: EGFR mutations in non-small-cell lung cancer: Analysis of a large series of cases and development of a rapid and sensitive method for diagnostic screening with potential implications on pharmacologic treatment. J. Clin. Oncol. 23, 857-865 (2005).

81 Mori S, Sugahara K, Uemura A et al.: Rapid, simple, and accurate detection of K-ras mutations from body fluids using real-time PCR and DNA melting curve analysis. Lab. Med. 37, 286-289 (2006).

82 Di Fiore F, Charbonnier F, Lefebure B et al.: Clinical interest of KRAS mutation detection in blood for anti-EGFR therapies in metastatic colorectal cancer. Br. J. Cancer 99, 551-552 (2008).

83 Artale S, Sartore-Bianchi A, Veronese SM et al: : Mutations of KRAS and BRAF in primary and matched metastatic sites of colorectal cancer. J. Clin. Oncol. 26, 4217-4219 (2008).

84 Trombino S, Neri M, Puntoni R et al:: Mutations in $K$-ras codon 12 detected in plasma DNA are not an indicator of disease in patients with non-small cell lung cancer. Clin. Chem. 51, 1313-1314 (2005).
85 Plesec TP, Hunt JL: KRAS mutation testing in colorectal cancer. Adv. Anat. Pathol. 16, 196-203 (2009).

86 Van Krieken JH, Jung A, Kirchner T et al.: $K R A S$ mutation testing for predicting response to anti-EGFR therapy for colorectal carcinoma: proposal for an European quality assurance program. Virchows Arch. 453, 417-431 (2008).

87 Jimeno A, Messersmith WA, Hirsch FR, Franklin WA, Eckhardt SG: KRAS mutations and sensitivity to epidermal growth factor receptor inhibitors in colorectal cancer: practical application of patient selection. J. Clin. Oncol. 27, 1130-1136 (2009).

88 Amur S, Frueh FW, Lesko LJ, Huang S: Integration and use of biomarkers in drug development, regulation and clinic practice: a US regulatory perspective. Biomarkers Med. 2, 305-311 (2008).

89 Su YH, Song J, Wang Z et al.: Removal of high-molecular-weight DNA by carboxylated magnetic beads enhances the detection of mutated K-ras DNA in urine. Ann. NY Acad. Sci. 1137, 82-91 (2008).

90 Mckinzie PB, Parsons BL: Detection of rare $\mathrm{K}-R A S$ codon 12 mutations using allelespecific competitive blocker PCR. Mutat. Res. 517(1-2), 209-220 (2002).

91 Diehl F, Schmidt K, Choti MA et al.: Circulating mutant DNA to assess tumor dynamics. Nat. Med. 14, 985-990 (2008).

92 Chen CY, Shiesh SC, Wu SJ: Rapid detection of K-ras mutations in bile by peptide nucleic acid-mediated PCR clamping and melting curve analysis: comparison with restriction fragment length polymorphism analysis. Clin. Chem. 50, 481-489 (2004).

93 Luthra R, Medeiros LJ: Isothermal multiple displacement amplification: a highly reliable approach for generating unlimited high molecular weight genomic DNA from clinical specimens. J. Mol. Diagn. 6, 236-242 (2004).

94 Chen YF, Wang JY, Wu CH, Chen FM, Cheng TL, Lin SR: Detection of circulating cancer cells with K-ras oncogene using membrane array. Cancer Lett. 229, 115-122 (2005).

95 Li J, Wang L, Mamon H, Kulke MH, Berbeco R, Makrigiorgos GM: Replacing PCR with COLD-PCR enriches variant DNA sequences and redefines the sensitivity of genetic testing. Nat. Med. 14, 579-584 (2008).

96 Chen YL, Chang YS, Chang JG, Wu SM: Genotyping of K-ras codons 12 and 13 mutations in colorectal cancer by capillary electrophoresis. J. Chromatography A 1216, 5147-5154 (2009).

97 Zhu D, Xing D, Tang Y, Zhang L: A novel mutant allele specific amplification and electrochemiluminescence method for the detection of point mutation in clinical samples. Biosensors Bioelectronics 24, 3306-3310 (2009).

98 Tol J, Koopman M, Cats A et al.: Chemotherapy, bevacizumab, and cetuximab in metastatic colorectal cancer. N. Engl. J. Med. 360, 563-572 (2009)

99 Bokemeyer C, Bondarenko I, Makhson A et al:: Fluorouracil, leucovorin, and oxaliplatin with and without cetuximab in the first-line treatment of metastatic colorectal cancer. J. Clin. Oncol. 27, 663-671 (2009).

100 Hecht JR, Mitchell E, Chidiac T et al.: A randomized Phase IIIB trial of chemotherapy, bevacizumab, and panitumumab compared with chemotherapy and bevacizumab alone for metastatic colorectal cancer. J. Clin. Oncol. 27, 672-680 (2009).

\section{Websites}

201 Catalogue of Somatic Mutations in Cancer. www.sanger.ac.uk/genetics/CGP/cosmic/

- A valuable resource for quick retrieval of pertinent information regarding the propensity for particular tumor types to carry particular tumor-associated mutations, includes data for many different oncogenes and tumor suppressor genes

202 College of American Pathologists Perspectives on Emerging Technology (POET) report. www.cap.org/apps/docs/committees/ technology/KRAS.pdf

203 Draft guidance for industry, clinical laboratories, and FDA staff on in vitro diagnostic multivariate index assays. www.fda.gov/cdrh/oivd/guidance/1610.pdf 